\title{
A Deep Intronic Variant Activates a Pseudoexon in the MTM1 Gene in a Family with X-Linked Myotubular Myopathy
}

\author{
Jamie Fitzgerald ${ }^{a, b, d} \quad$ Cori Feist $^{c} \quad$ Paula Dietz $^{a} \quad$ Stephen Moore $^{d, e} \quad$ Donald Basel ${ }^{d, f}$ \\ aDepartment of Orthopedic Surgery, Bone and Joint Center, Henry Ford Hospital System, Detroit, MI, USA; ${ }^{b}$ Department of \\ Orthopedics and Rehabilitation, Oregon Health and Science University, Portland, OR, USA; 'Department of Obstetrics and \\ Gynecology, Oregon Health and Science University, Portland, OR, USA; ${ }^{\mathrm{d} D e p a r t m e n t ~ o f ~ M o l e c u l a r ~ a n d ~ M e d i c a l ~ G e n e t i c s, ~}$ \\ Oregon Health and Science University, Portland, OR, USA; ${ }^{\mathrm{e} K n i g h t ~ D i a g n o s t i c ~ L a b o r a t o r i e s, ~ O r e g o n ~ H e a l t h ~ a n d ~ S c i e n c e ~}$ \\ University, Portland, OR, USA; 'Department of Genetics, Medical College of Wisconsin, Milwaukee, WI, USA
}

\section{Keywords}

Deep intron · Exonization · MTM1 - Pseudoexon · RNA splicing $\cdot$ XLMTM

\section{Abstract}

We report a novel intronic variant in the MTM1 gene in 4 males in a family with severe $\mathrm{X}$-linked myotubular myopathy. The $A>G$ variant in deep intronic space activates a cryptic $5^{\prime}$ donor splice site resulting in the inclusion of a 48-bp pseudoexon into the mature MTM1 mRNA. The variant is present in all affected males, absent in unaffected males, and heterozygous in the mother of the affected males. The included intronic sequence contains a premature stop codon, and experiments using a translational inhibitor indicate that the mutant mRNAs undergo nonsense-mediated decay. We conclude that affected males produce no, or low, levels of MTM1 mRNA likely leading to a significant reduction of myotubularin-1 protein resulting in the severe neonatal myopathy present in this family. The study highlights the need to consider noncoding variants in genomic screening in families with X-linked myotubular myopathy.

(c) 2020 The Author(s)

Published by S. Karger AG, Basel

karger@karger.com www.karger.com/msy

Karger $\stackrel{\text { ' }}{=}$

马OPEN ACCESS
(C) 2020 The Author(s)

Published by S. Karger AG, Basel

This is an Open Access article licensed under the Creative Commons Attribution-NonCommercial-4.0 International License (CC BY-NC) (http://www.karger.com/Services/OpenAccessLicense), applicable to the online version of the article only. Usage and distribution for commercial purposes requires written permission.

\section{Introduction}

RNA splicing is a fundamental and tightly regulated process that directs the production of multiple protein isoforms from a single gene by removing introns and combining different exons. Pseudoexons are intronic sequences, sometimes far from exons, that have the basic consensus sequences at the $5^{\prime}$ and $3^{\prime}$ splice sites but are not normally spliced into mature mRNA [Buratti et al., 2007; Dhir and Buratti, 2010; Dhir et al., 2010]. From a human disease perspective, many pseudoexon intronic sequences are poised on the brink of becoming exons but require an additional sequence change to activate cryptic splice sites. When these mutations occur and the cryptic splice sites are activated, pseudoexons can be "spliced-in" to become "pathogenic pseudoexons" [Dhir and Buratti, 2010]. De novo mutations that activate pseudoexons have been reported in approximately 60 genes, according to the DBASS3 and DBASS5 aberrant splice site databases (http://www.dbass.org.uk) [Buratti et al., 2011].

In recent years, RNA-seq approaches, where the entire transcriptome is sequenced, have been successful at identifying intronic variants that result in aberrant splicing in

Jamie Fitzgerald

Department of Orthopedic Surgery, Bone and Joint Center Henry Ford Hospital System

6135 Woodward Ave, Detroit MI 48202 (USA) jfitzge2@hfhs.org 
musculoskeletal disease [Cummings et al., 2017; Hamanaka et al., 2019]. These studies reveal that as a mutation class, intronic variants are a relatively common contribution to human disease and RNA-seq is emerging as a powerful diagnostic tool for mutation detection in musculoskeletal disease [Gonorazky et al., 2019].

X-linked myotubular myopathy (XLMTM; OMIM 310400 ) is the most common of the centronuclear myopathies, and the only one to have an X-linked inheritance pattern. Patients show generalized muscle weakness and respiratory insufficiency that often leads to death in the neonatal period or childhood. MTM1 is a 14-exon gene located in the distal long arm of the $\mathrm{X}$ chromosome, band Xq28 (NM_000252.2). The MTM1 gene product, myotubularin-1, functions as a 3-phosphoinositide phosphatase involved in phosphoinositol signaling and is required for skeletal muscle cell differentiation [Spiro et al., 1966; van Wijngaarden et al., 1969]. More than 400 MTM1 mutations in XLMTM have been described to date. Pathogenic variants that result in the loss of myotubularin-1 protein are frequently associated with demise as a result of respiratory insufficiency, and missense mutations are generally associated with milder forms and prolonged survival [Laporte et al., 2000].

Here, we report a novel pathogenic pseudoexon variant in the MTM1 gene in a family with a severe form of XLMTM.

\section{Materials and Methods}

Cell Culture and Cycloheximide Treatment

Primary skin fibroblasts were established from patient skin biopsies and maintained in DMEM/10\% fetal calf serum supplemented with $100 \mathrm{U} / \mathrm{mL}$ penicillin and $100 \mu \mathrm{g} / \mathrm{mL}$ streptomycin (Gibco). To stabilize proteins containing premature stop codons, cultures were treated for $6 \mathrm{~h}$ with $100 \mu \mathrm{g} / \mathrm{mL}$ cycloheximide and RNA isolated for RT-PCR [Bateman et al., 1999]. Control skin fibroblasts were derived from individuals unrelated to the XLMTM1 family.

\section{RT-PCR}

Total RNA was isolated from cultures using the RNeasy kit (Qiagen), quantitated and $1 \mu \mathrm{g}$ of RNA reverse transcribed into cDNA using the iScript kit (Bio-Rad). Three overlapping MTM1 cDNAs were amplified using 3 pairs of cDNA primers from Tosch et al. [2010] using the PrimeSTAR GxL kit (Takara). Primers are F1/R1 (ATGGCTTCTGCATCAACTTC/TGGAATTCGATTTCGGGAC) for fragment 1 (678 nt), F2/R2 (GTTCCGTATCGTGCCTCAG/GGAGAACGGTCAGCATCGG) for fragment 2 (698nt) andF3/R3 (AGAATGGATAAGTTTTGGAC/TTATTTCGAGCTCTAATGCG) for fragment 3 (622 nt).

For quantitative PCR, cDNAs were diluted $1 / 20$ and amplification conducted using SYBR Green (Applied Biosystems) with the
F3/R3 primer set in quadruplicate under conditions of maximal efficiency on an ABI2400 quantitative thermocycler. Data analysis was carried out using the comparative $\Delta \mathrm{Ct}$ method using the L32 ribosomal gene as a housekeeping gene.

\section{Results}

\section{Clinical Description}

The family was identified in 2005 when a neonatal male (IV-1 in Fig. 1) presented to the neonatal intensive care unit with lack of respiratory effort and severe hypotonia. He was born to a 16-year-old G1P0 woman at 36 weeks' gestation. Her pregnancy was complicated by severe polyhydramnios. He required mechanical ventilation and underwent several laboratory evaluations including normal plasma creatine kinase levels, normal methylation for Prader-Willi syndrome, 2 copies of $S M N 1$, normal testing for myotonic dystrophy type 1 , and normal very longchain fatty acids. Muscle biopsy at day of life 7 was concerning for a central nuclear myopathy. Blood was sent for MTM1 gene sequencing, which did not reveal any sequence changes in the 14 coding exons or the $5^{\prime}$-untranslated region of exon 1 . He was transitioned to comfort care and died at day of life 25. No autopsy was performed. His maternal cousin (IV-6), also male, was born in 2009 with a similar clinical presentation including severe polyhydramnios precipitating delivery at $35 \mathrm{w} 5 \mathrm{~d}$ gestation. The baby was transferred to the neonatal intensive care unit at delivery with hypotonia and absent respiratory effort requiring mechanical ventilation. Muscle biopsy at day of life 4 revealed relative smallness of type 1 and $2 \mathrm{~b}$ fibers but no discernible features of a centronuclear myopathy. He was transitioned to comfort care and died at 11 days of age. Autopsy was performed but of limited clinical usefulness.

In 2011, another male (IV-2) was born to the obligate carrier female (III-1), with the same condition. He was delivered at 35 weeks' gestation by repeat Cesarean section for severe polyhydramnios. At birth, he had no spontaneous respirations and required aggressive resuscitation. He was intubated, but after no response to chest compressions, fluid bolus, and epinephrine resuscitation efforts were discontinued. He died 4 min later. Blood was sent for karyotyping (46,XY), but no other genetic testing or autopsy was performed. In 2016, a third affected son (IV-3) was born to III-1 by repeat Cesarean section at 37 weeks for polyhydramnios. This male was intubated at birth for no respiratory effort but was able to transition to continuous positive airway pressure at 2 days of age. For unclear reasons, this child appeared less severely affected and was 


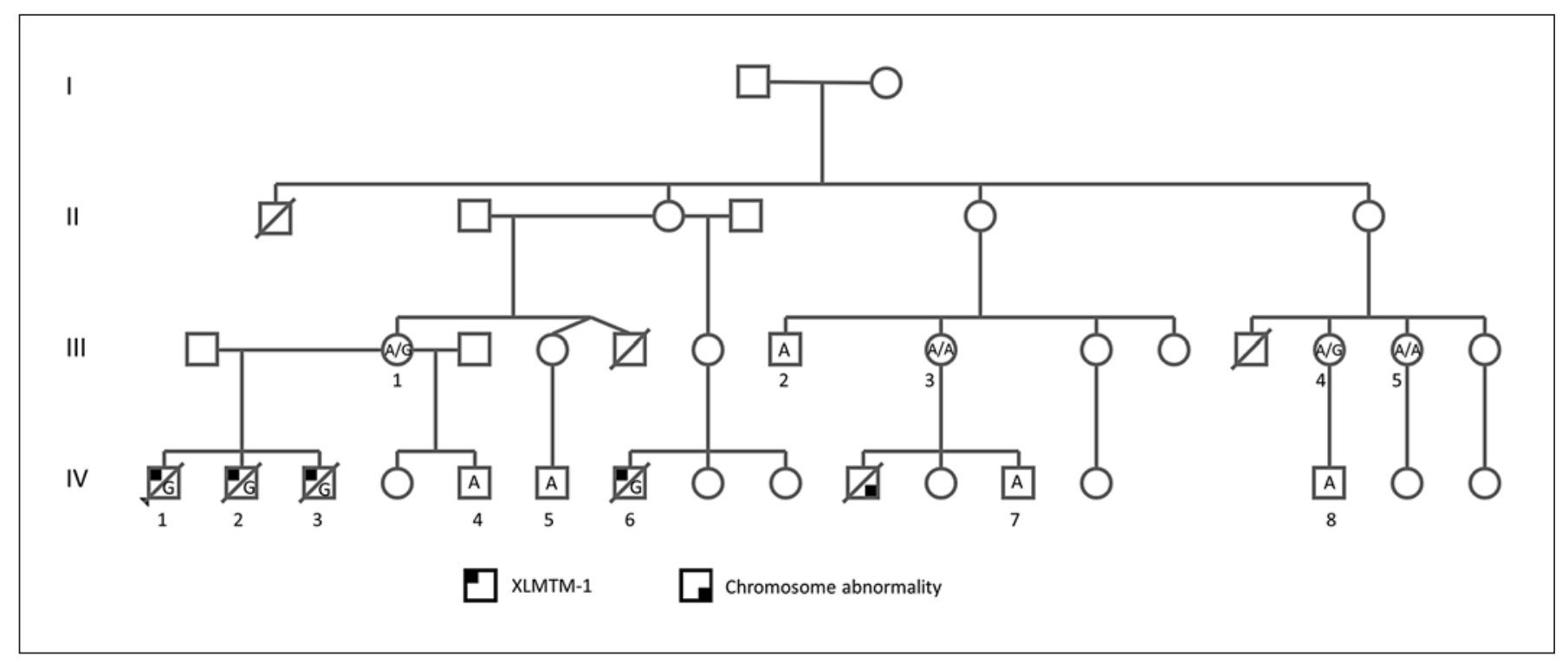

Fig. 1. Pedigree of extended family with XLMTM1.

able to transition to bilevel positive airway pressure at 14 weeks of life. He underwent G-J tube placement at 11 weeks of life as well as various diagnostic studies. Testing for SMN1-related spinal muscular atrophy, Prader-Willi syndrome, myotonic dystrophy type 1 , and trio whole exome sequencing in a CLIA/CAP approved laboratory was non-diagnostic. Muscle biopsy at 11 weeks of life was suggestive of centronuclear myopathy. This child was evaluated throughout his admission by pediatric neuromuscular specialists and placed on mestinon $1 \mathrm{mg} / \mathrm{kg} /$ dose TID at 15 weeks of life. He was discharged from the neonatal intensive care unit at 18 weeks of life ( 55 weeks corrected gestational age). He died suddenly of cardiorespiratory arrest at 1 year and 11 days of life. Pedigree of the extended family is shown in Figure 1. The mother (III-1) of 3 affected males (IV-1, IV-2, and IV-3) appears to be mildly affected with EMG evidence of mild isolated axial myopathy in the thoracic paraspinal muscles. Spirometry indicates a $35 \%$ decrease in forced vital capacity from standing to supine, which is suggestive of diaphragmatic weakness. The other carrier female identified was not clinically examined.

\section{Identification of Intronic Variant}

Since the proband was considered to have an X-linked myopathy similar to XLMTM1, the coding region of the MTM1 gene was sequenced manually independent of the clinical sequencing. This analysis failed to identify a mutation in the MTM1 exons and intron-exon boundaries. Since it is emerging that a significant fraction of genetic disease is due to mutations in noncoding regions, RT-PCR was conducted on RNA isolated from fibroblasts from one of the affected males (Fig. 2a). One fragment (PCR3) produced a larger than expected RT-PCR product in the patient compared to control cDNA suggesting the inclusion of additional sequence in the mature mRNA. Sequencing the PCR3 product revealed that the additional sequence was a 48-bp stretch of intronic sequence spanning chrX:149831277_149831324 in MTM1 intron 13. Sequencing of genomic DNA flanking the included sequence revealed an $A>G$ substitution 5 bases downstream of the end of the included sequence at chrX:149831329A $>\mathrm{G}$ c.1468-577 (MTM1, NM_000252.2) (Fig. 2b). This variant is $2,371 \mathrm{bp}$ downstream from exon 13 and $577 \mathrm{bp}$ from the start of exon 14. The variant is unique and not present in the gnom $A D$ variant database, and no ESTs containing the included intron have been described to date. Genotyping 13 family members demonstrated that all the affected (individuals IV $-1,-2,-3$, and -6 ), but none of the unaffected males (IV $-4,-5,-7$, and -8 ), carry the variant. The mother (III-1) of 3 affected males is heterozygous for the variant, as expected, as is the mother (III-4) of an unaffected boy (IV-8). Sequencing chromatograms of the carrier mother, affected and unaffected sons showing their expected genotypes, is shown in Figure 2c.

\section{Variant Results in Altered Splicing}

In silico support for altered RNA splicing comes from the Human Splicing Finder splice prediction software 


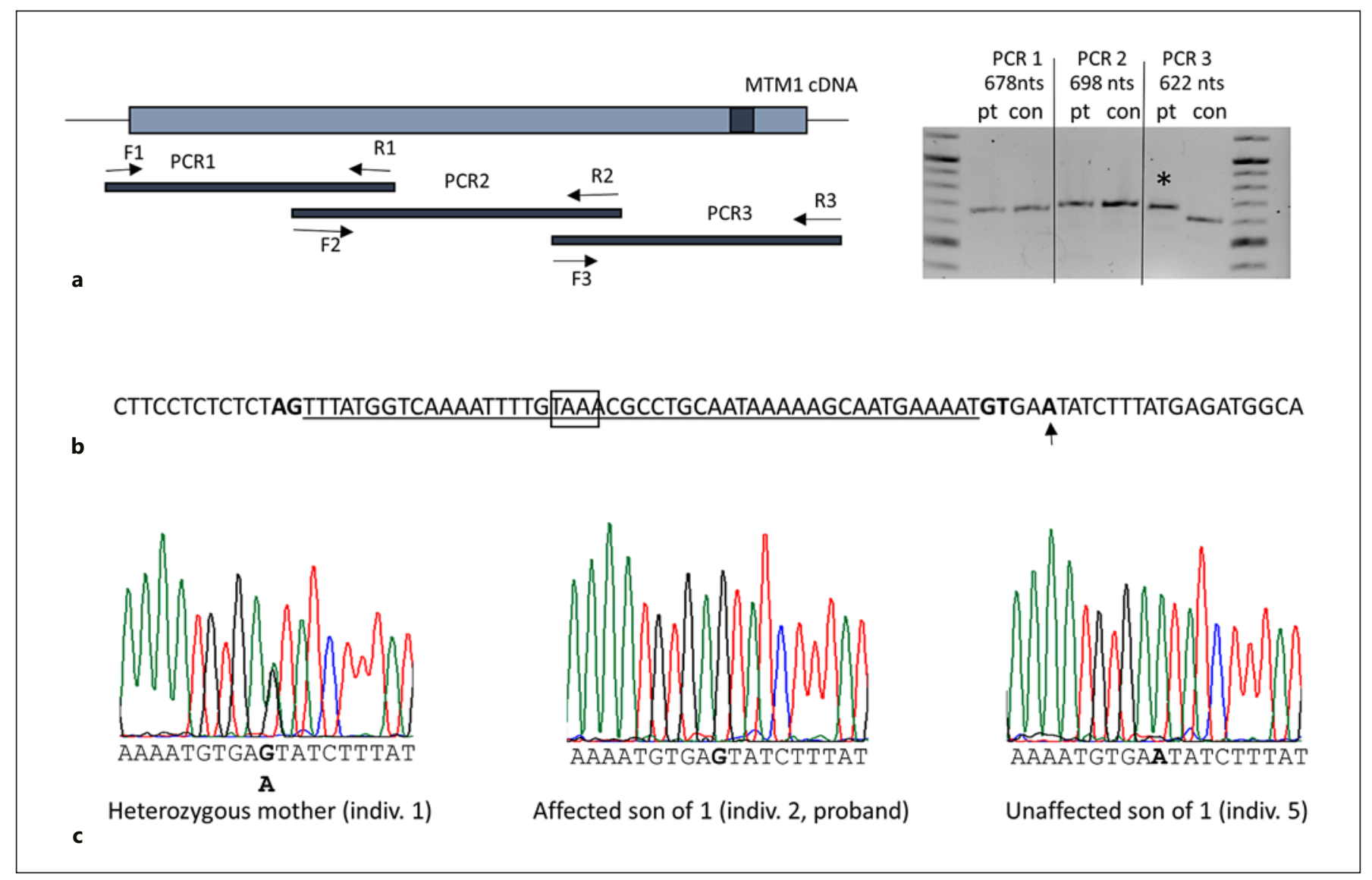

Fig. 2. Identification of a pathogenic intron variant in MTM1. a Aberrant MTM1 RNA splicing in the XLMTM1 family. Three overlapping RT-PCR fragments encompassing the MTM1 cDNA were designed based on published primers [Tosch et al., 2010]. RNA from patient fibroblasts was isolated, reverse transcribed into cDNA and subjected to PCR using the 3 primer sets - F1/R1, F2/ $\mathrm{R} 2$, and F3/R3. The right panel is a DNA agarose gel showing 3 RT-PCR products from patient (pt) and control (con) fibroblasts. PCR3 is larger in patient compared to control suggesting aberrant splicing and the inclusion of intronic sequence in the mature mRNA (pseudoexon). This is indicated by the black box. The expected size of PCR amplicons is shown above gel. Marker is 100-bp ladder. b Genomic context of MTM1 mutation. The PCR3 band was sequenced and found to contain 48 bp of MTM1 intron 13 (underlined). Sequencing of genomic DNA in this intronic region showed the presence of a unique $A>G$ substitution (in bold and

(http://www.umd.be/HSF3/HSF.shtml) [Desmet et al., 2009; Flicek et al., 2013]. The G $>$ A variant increases the MaxEnt splicing score from -0.4 to +7.8 . With the threshold for splicing set at 3 , the wild-type sequence is not predicted to be spliced, and the mutant sequence is strongly predicted to be spliced (Fig. 3a). It is also noted that substitution of $\mathrm{G}$ for $A$ at the +5 position creates a consensus sequence for a U1-snRNP splicing factor binding (A-G-G-U-A/G-A-G). indicated by an arrow). The variant is $5 \mathrm{bp}$ downstream of the $5^{\prime}$ cryptic splice site acceptor that is not normally activated. Canonical AG $3^{\prime}$ splice donor and GT $5^{\prime}$ splice acceptor sites that define the boundaries of the pseudoexon are in bold. The in-frame stop codon (TAA) is boxed. c Genotyping family members for the $A>G$ variant in intron 13. Family members were genotyped by sequencing across the genomic region containing the variant. A sequencing chromatogram shows that the carrier female (III-1) and mother to 3 of the affected males is heterozygous for the variant (left). Her affected son and proband (IV-1) is hemizygous for the G allele (middle), and an unaffected son (IV-4) is hemizygous for the A allele (right). DNA sequence of the variant is shown below each chromatogram, and the variant alleles are in bold. Genotyping the remaining extended family members show that all affected males carry the $\mathrm{G}$ variant and all unaffected males the $\mathrm{A}$ variant.

Collectively, the experimental and prediction data indicate that the $A>G$ variant activates a cryptic 5 ' donor splice site in a pseudoexon in intron 13 leading to inclusion of intronic sequence in affected individuals. In unaffected individuals, the wild-type sequence is not sufficient to activate splicing of the pseudoexon and a normal splicing pattern is maintained. The new splice site acts in concert with the normal downstream $3^{\prime}$ acceptor splice site adjacent to 


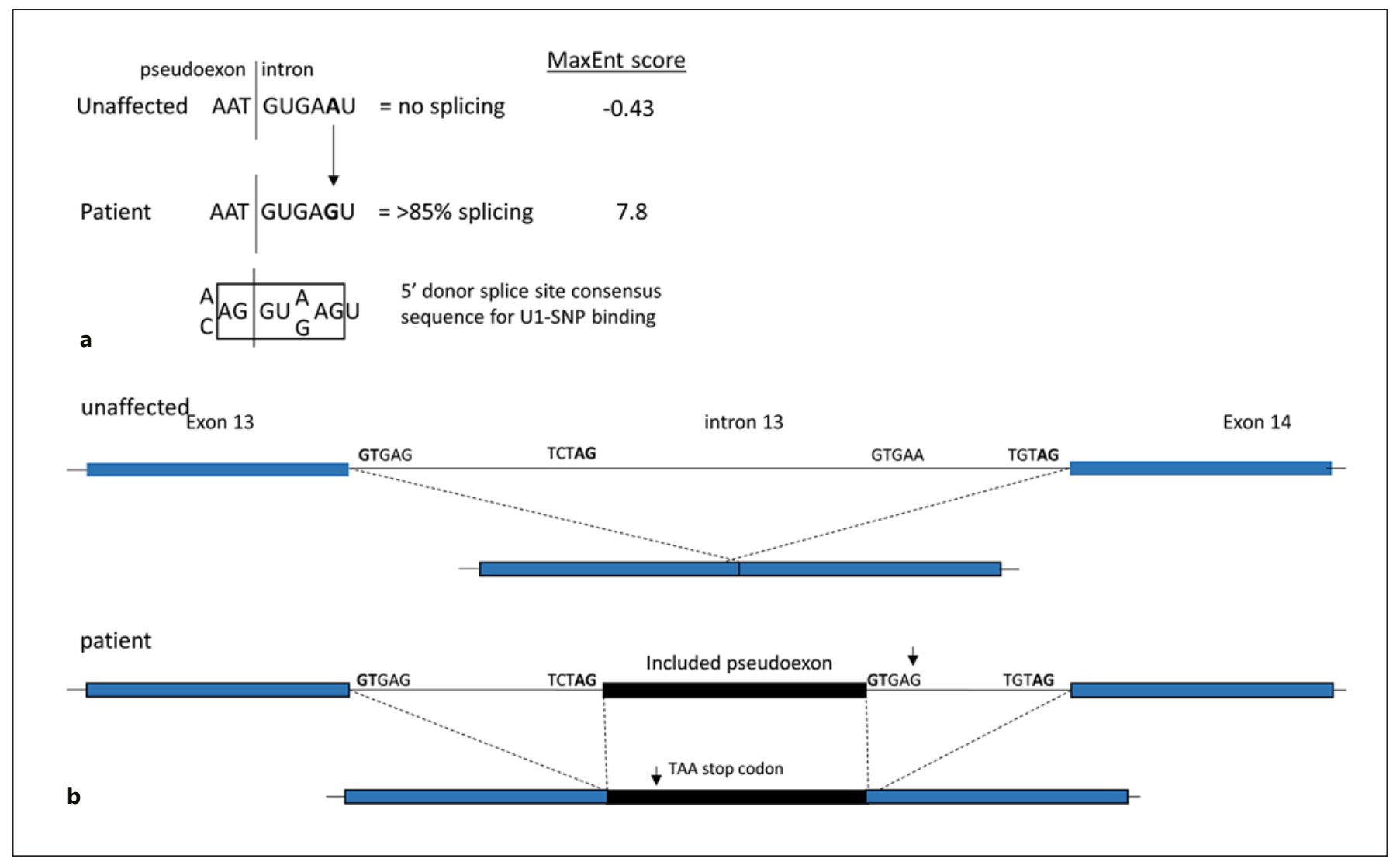

Fig. 3. The $A>G$ variant activates a cryptic $5^{\prime}$ splice site. a In silico analysis of altered splice site. The $A>G$ nucleotide change converts a pseudoexon, which is not normally spliced, into an "included pseudoexon". Densitometric analysis of RT-PCR products indicates that $85 \%$ of $M T M 1$ transcripts contain the included pseduoexon. The $5^{\prime}$ splice site consensus sequence is shown below and the U1-snRNP binding site is boxed. Analysis using the MaxEnt tool of the Human Splicing Finder software predicts that the mutant sequence increases splicing efficiency. Since the score of the wildtype sequence is below the threshold of 3 , it is not predicted to be spliced. In contrast, with a score of 7.8 , the mutant sequence is

exon 14. Similarly, the existing $5^{\prime}$ acceptor splice site near the start of the pseudoexon is used with the $5^{\prime}$ donor splice site adjacent to the upstream exon 13 (Fig. 3b). The net result of the mutation is the preferential inclusion of the pseudoexon in individuals with the $G$ variant.

\section{Decay of the Mutant $m R N A$}

The included 48-bp pseudoexon sequence contains an in-frame TAA stop codon at chrX:149831295_149831297, suggesting that the mutant mRNA may undergo nonsense-mediated mRNA decay. This was confirmed by RTPCR across the pseudoexon region in the proband and an affected brother that cells were available for (Fig. 4a). The strongly predicted to be spliced. b Schematic showing splicing pattern in patient and unaffected individuals due to $A>G$ mutation. Normal splicing pattern in unaffected individuals (top) with exclusion of intron 13 and splicing together of exons and 14. Intron splice site acceptor and donor sequences are shown. Splicing pattern of affected individuals (bottom) showing the effect of $A>G$ mutation (arrow). Activation of $5^{\prime}$ donor site (GTGAG) in pseudoexon also results in preferential use of existing $3^{\prime}$ acceptor site (TCTAG) at $5^{\prime}$ end of pseudoexon to effect splicing of 48-bp pseudoexon sequence. Approximate location of TAA stop codon in included sequence is indicated. pseudoexon band is much fainter in the affected sibling than in control fibroblasts. When patient fibroblasts were incubated with the translational inhibitor, cycloheximide, degradation of the mutant allele was halted, providing further evidence that the mutant allele undergoes nonsensemediated mRNA decay. Quantitative PCR analysis reveals that the mutant allele is stabilized 7-fold in the presence of cycloheximide (Fig. 4b). Direct demonstration of the loss of myotubularin-1 protein was not possible because myotubularin-1 protein was not detectable in normal human fibroblasts using 3 commercial antibodies and polyclonal antisera produced in-house (data not shown). This is likely due to the low levels of baseline myotubulatin-1 protein 


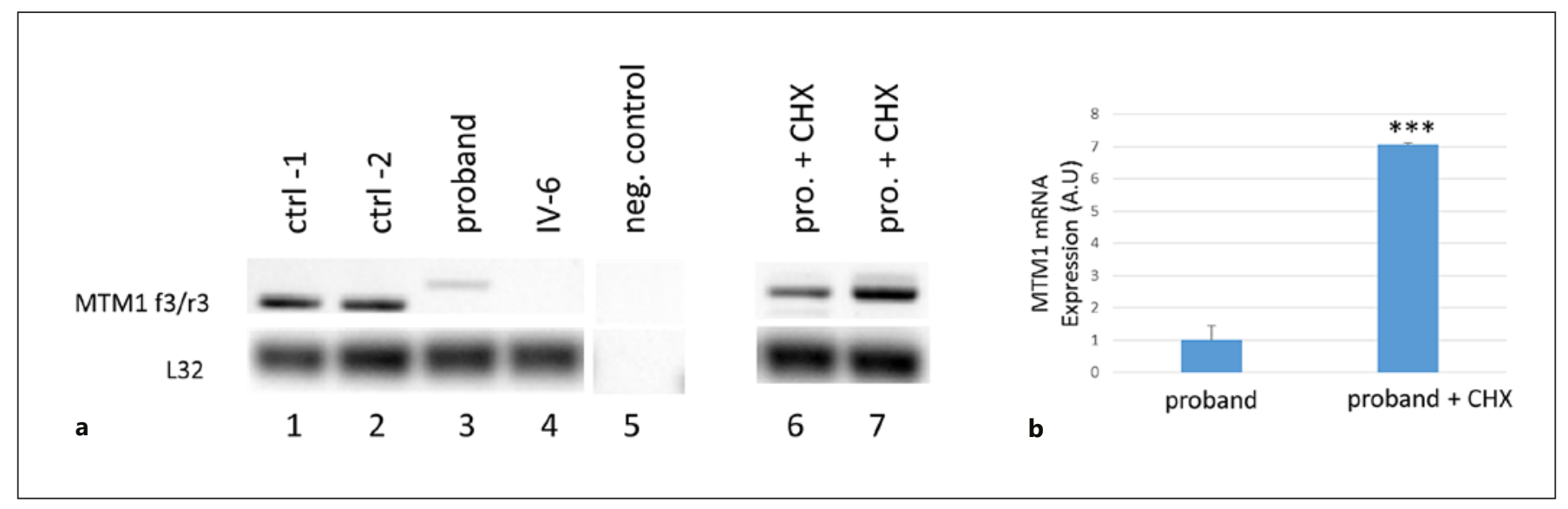

Fig. 4. mRNA for mutant MTM1 allele undergoes nonsense-mediated mRNA decay.a RT-PCR for normal and mutant mRNA. cDNA samples from 2 control fibroblast cell lines (lanes 1 and 2), the proband (IV-1, lane 3), and an affected cousin (IV-6, lane 4) were amplified using the MTM1 F3/R3 primers. No template negative control is shown in lane 5. Virtually no normally spliced MTM1 mRNA is detected in patient cells compared to control cells, and a low level of mutant mRNA is present in patient cells indicating that the mutant allele is degraded. In a separate experi-

in skin fibroblasts. However, since the mutant allele is substantially degraded, it is reasonable to assume that affected males produce no, or low levels, of the myotubularin-1 protein, while carrier females produce sufficient myotubularin for survival.

\section{Discussion}

We report a novel variant in intron 13 of the MTM1 genes in a family with XLMTM. The mutation results in the inclusion of a pathogenic pseudoexon that leads to decay of the mutant mRNA. It is predicted that affected males are essentially null for myotubularin-1, the MTM1 gene product. The mutation is severe and 3 of the 4 affected males died in the neonatal period due to respiratory insufficiency while the fourth child survived for 12 months.

Tosch et al. [2010] estimated that intronic mutations account for approximately $19 \%$ of the MTM1 mutations with the majority of these being intron-exon boundaries which are readily detectable by routine genomic DNA sequencing. This group employed a screen specifically to detect intronic mutations in the MTM1 gene and, in addition to identifying several novel intron-exon boundary mutations, identified the first pseudoexon mutation in MTM1 [Tosch et al., 2010]. RT-PCR and sequencing revealed 94 bp of included intron sequence in intron 7 . The mutation ment, proband samples were cultured in the absence (lane 6) and presence (lanes 7) of cycloheximide. The mutant mRNA is stabilized by cycloheximide. L32 ribosomal mRNA is a housekeeping gene used as a control for cDNA. b Quantitative PCR (qPCR) analysis of cycloheximide-treated patient cells. qPCR was conducted on cDNA isolated from proband cells treated with and without the translation inhibitor cycloheximide in quadruplicate. Cycloheximide treatment results in $7 \times$ more mutant mRNA compared to untreated cells. ${ }^{* * *} p=0.0004$, Student $t$ test. Error bars are SDs. created a functional splice acceptor site that when used together with an existing splice donor site resulted in exonization, leading to nonsense-mediated mRNA decay and loss of myotubilarin-1 protein. Our report is the second of these types of mutations in severe XLMTM1 and highlights the importance of deep intronic mutations in the pathogenesis of XLMTM1 and the need to include screening strategies when looking for MTM1 mutations.

From a treatment perspective, the observation that the disorder is nonlethal in carrier females suggests that a modest increase in myotubularin levels may be therapeutically useful, although it remains to be determined how much myotubularin-1 is required for survival to adulthood. Further complicating our understanding of how much myotubularin-1 is required for normal development is the presence of muscle weakness in the probands' mother, suggesting that she may be mildly affected. Phenotypic variability in ambulatory status and muscle weakness in carrier females with MTM1 mutations has been reported previously [Cocanougher et al., 2019]. A more detailed clinical analysis of her and the other carrier females in the family will be required to confirm the presence of mild XLMTM1 and whether there is intra-familial variability. We observed differences in the extent of mutant mRNA degradation (see Fig. 4a) between affected individuals, suggesting that the extent of cryptic splice site activation may differ between individuals. This may 
explain why one of the 4 affected males in the family survived past one year and leads us to speculate that there is a threshold for myotubularin-1 levels such that the male that survived the neonatal period had less aberrant splicing and a higher incidence of normal splicing.

One therapeutic approach to increase the levels of myotubularin-1 could be to correct normal splicing in MTM1 using blocking antisense oligonucleotides. The suppression of pseudoexon inclusion using oligonucleotides has been demonstrated in patient cells containing an intronic mutation in the COL6A1 gene [Bolduc et al., 2019] and appears to be a promising therapeutic strategy.

In summary, we described an intronic mutation which leads to $5^{\prime}$ splice site activation and the inappropriate inclusion of a segment of intron sequence into the mature mRNA. This leads to an in-frame premature stop codon and decay of the mutant mRNA which is catastrophic for the affected males in the family resulting in a lethal phenotype. Our study highlights the need to consider noncoding mutations in mutation screening in XLMTM1 families and suggests that RNAseq would be a useful diagnostic tool for the capture of this class of mutations.

\section{Acknowledgements}

We would like to thank the family who generously provided samples for this study.

\section{Statement of Ethics}

This research was conducted ethically in accordance with the World Medical Association Declaration of Helsinki. Subjects provided their written informed consent and that the study protocol was approved by the Institutional Review Boards of Oregon Health and Science University (protocol \#6758) and Henry Ford Hospital System (protocol \#10967).

\section{Conflict of Interest Statement}

The authors have no conflicts of interest to declare.

\section{Funding Sources}

Salary support to J.F. was provided, in part, by the National Institute of Arthritis and Musculoskeletal and Skin Diseases of the National Institutes of Health under Award Number R01AR055957.

\section{Author Contributions}

J.F. and D.B. were involved in the overall design of the project and conducted genetic analyses of samples. C.F. was the genetic counselor that liaised with the family and collected patient samples. P.D. conducted technical analyses. S.M. provided patient cell lines for the project. J.F., D.B., C.F., and S.M. wrote and edited the manuscript.

\section{References}

Bateman JF, Freddi S, Lamandé SR, Byers P, Nasioulas S, Douglas J, et al. Reliable and sensitive detection of premature termination mutations using a protein truncation test designed to overcome problems of nonsense-mediated mRNA instability. Hum Mutat. 1999;13(4):311-7..

Bolduc V, Foley AR, Solomon-Degefa H, Sarathy A, Donkervoort S, Hu Y, et al. A recurrent COL6A1 pseudoexon insertion causes muscular dystrophy and is effectively targeted by splice-correction therapies. JCI Insight. 2019; 4(6):e124403.

Buratti E, Dhir A, Lewandowska MA, Baralle FE. RNA structure is a key regulatory element in pathological ATM and CFTR pseudoexon inclusion events. Nucleic Acids Res. 2007; 35(13):4369-83..

Buratti E, Chivers M, Hwang G, Vorechovsky I. DBASS3 and DBASS5: databases of aberrant 3'- and 5'-splice sites. Nucleic Acids Res. 2011; 39(Database issue):D86-91..
Cocanougher BT, Flynn L, Yun P, Jain M, Waite M, Vasavada R, et al. Adult MTM1-related myopathy carriers: classification based on deep phenotyping. Neurology. 2019;93(16): e1535-e1542..

Cummings BB, Marshall JL, Tukiainen T, Lek M, Donkervoort S, Foley AR, et al. Improving genetic diagnosis in Mendelian disease with transcriptome sequencing. Sci Transl Med. 2017;9(386):eaal5209.

Desmet FO, Hamroun D, Lalande M, CollodBéroud G, Claustres M, Béroud C. Human Splicing Finder: an online bioinformatics tool to predict splicing signals. Nucleic Acids Res. 2009;37(9):e67..

Dhir A, Buratti E. Alternative splicing: role of pseudoexons in human disease and potential therapeutic strategies. FEBS J. 2010;277(4): 841-55..

Dhir A, Buratti E, van Santen MA, Lührmann R, Baralle FE. The intronic splicing code: multiple factors involved in ATM pseudoexon definition. EMBO J. 2010;29(4):749-60..

Flicek P, Ahmed I, Amode MR, Barrell D, Beal K, Brent S, et al. Ensembl 2013. Nucleic Acids Res. 2013;41(Database issue):D48-55..
Gonorazky HD, Naumenko S, Ramani AK, Nelakuditi V, Mashouri P, Wang P, et al. Expanding the boundaries of RNA sequencing as a diagnostic tool for rare Mendelian disease. Am J Hum Genet. 2019;104(5): 1007.

Hamanaka K, Miyatake S, Koshimizu E, Tsurusaki Y, Mitsuhashi S, Iwama K, et al. RNA sequencing solved the most common but unrecognized NEB pathogenic variant in Japanese nemaline myopathy. Genet Med. 2019; 21(7):1629-38.

Laporte J, Biancalana V, Tanner SM, Kress W, Schneider V, Wallgren-Pettersson C, et al. MTM1 mutations in X-linked myotubular myopathy. Hum Mutat. 2000;15(5):393-409..

Spiro AJ, Shy GM, Gonatas NK. Myotubular myopathy. Persistence of fetal muscle in an adolescent boy. Arch Neurol. 1966;14(1):1-14..

Tosch V, Vasli N, Kretz C, Nicot AS, Gasnier C, Dondaine $\mathrm{N}$, et al. Novel molecular diagnostic approaches for X-linked centronuclear (myotubular) myopathy reveal intronic mutations. Neuromuscul Disord. 2010;20(6):375-81.

van Wijngaarden GK, Fleury P, Bethlem J, Hugo Meijer AEF. Familial "myotubular" myopathy. Neurology. 1969;19(9):901-8.. 